\title{
Gearbox Fault Features Extraction Using Vibration Measurements and Novel Adaptive Filtering Scheme
}

\author{
Ghalib R. Ibrahim ${ }^{1}$ and A. Albarbar ${ }^{2}$ \\ ${ }^{1}$ Mechanical Engineering Department, College of Engineering, University of Anbar, Anbar, Iraq \\ ${ }^{2}$ Advanced Industrial Diagnostic Centre, School of Engineering, Manchester Metropolitan University, Manchester M1 5GD, UK
}

Correspondence should be addressed to A. Albarbar, a.albarbar@mmu.ac.uk

Received 2 April 2012; Revised 29 May 2012; Accepted 11 June 2012

Academic Editor: Akira Ikuta

Copyright ( 2012 G. R. Ibrahim and A. Albarbar. This is an open access article distributed under the Creative Commons Attribution License, which permits unrestricted use, distribution, and reproduction in any medium, provided the original work is properly cited.

\begin{abstract}
Vibration signals measured from a gearbox are complex multicomponent signals, generated by tooth meshing, gear shaft rotation, gearbox resonance vibration signatures, and a substantial amount of noise. This paper presents a novel scheme for extracting gearbox fault features using adaptive filtering techniques for enhancing condition features, meshing frequency sidebands. A modified least mean square (LMS) algorithm is examined and validated using only one accelerometer, instead of using two accelerometers in traditional arrangement, as the main signal and a desired signal is artificially generated from the measured shaft speed and gear meshing frequencies. The proposed scheme is applied to a signal simulated from gearbox frequencies with a numerous values of step size. Findings confirm that $10^{-5}$ step size invariably produces more accurate results and there has been a substantial improvement in signal clarity (better signal-to-noise ratio), which makes meshing frequency sidebands more discernible. The developed scheme is validated via a number of experiments carried out using two-stage helical gearbox for a healthy pair of gears and a pair suffering from a tooth breakage with severity fault 1 (25\% tooth removal) and fault 2 (50\% tooth removal) under loads $(0 \%$, and $80 \%$ of the total load). The experimental results show remarkable improvements and enhance gear condition features. This paper illustrates that the new approach offers a more effective way to detect early faults.
\end{abstract}

\section{Introduction}

Fault detection techniques include many parameters which are based on time domain statistical analysis, frequency domain (fast Fourier transform), and time-frequency techniques to detect damage of the gearbox. Liao et al. [1] introduced a self-organizing feature maps (SOFM) network technique as a tool for gearbox CM. Gearbox vibration signals were captured under different operating conditions: normal, tooth cracked, and tooth broken. The results showed that the SOFM network gave a good representation for understanding the distribution of the vibration signal. Moreover, the growth of damage can early be monitored via the path of the image points for the feature data in two-dimensional space. He et al. [2] employed the kernel principal component analysis (KPCA) to extract nonlinear features from the time- and frequency-domains of the vibration signals. To classify the gearbox conditions, they constructed two different KPCA-based subspace structures. A fatigue test of an automobile transmission gearbox was carried out and the data set was analysed using the proposed method. The results illustrated that the KPCA features were better than PCA features in terms of clustering capability, and both the two KPCA-based subspace methods can be effectively applied to gearbox CM. Rafiee and Tse [3] used autocorrelation of continuous wavelet coefficients (CWT) to detect a fault in a gear. A motorcycle gearbox system was used under different operating conditions; normal gearbox (NG), slight-worn (SW), medium-worn (MW), and brokentooth gear (BT). Their conclusions were (a) autocorrelation CWT is a suitable method for feature extraction from nonstationary signals in gearbox for $\mathrm{CM}$, (b) the proposed technique could be used to monitor other vibration signals and diagnose faults in bearings. Zhou et al. [4] used the ensemble empirical mode decomposition (EEMD) method to diagnose localized damage of the feed-axis gearbox. 
Built-in position sensors such as motor encoders and linear scales with high resolution and high precision were employed in their study. Their results were that, (a) the EEMD method was cheaper to use as it was simpler and did not require an extra sensor, (b) the EEMD was efficient and effective in determining the problems in the system. Eftekharnejad and Mba [5] investigated the effectiveness of acoustic emission (AE) in identifying seeded defects in helical gears. The results identified the RMS levels from the acoustic signal are more capable of correctly identifying the seeded defects on helical gears than the vibration signals from an accelerometer mounted on the bearing pedestal. Saravanan et al. [6] presented a method that used ANN and proximal support vector machines (PSVM) to classify the fault in a gearbox. Statistical techniques were used to calculate Morlet wavelet coefficients and were fed as input for training the ANN and PSVM, and testing their relative efficiency in classifying the faults in a bevel gear box. The findings showed that the PSVM had an edge over ANN in classification of parameters. Albarbar et al. [7] presented adaptive filtering techniques to enhance diesel fuel-injector needle impact excitations contained within the air-borne acoustic signals. The results showed that the proposed technique was useful in enhancing other impulsive events within the airborne acoustic signals such as intake and exhaust valves operation and piston slap excitations. Recently, Ibrahim and Albarbar [8] presented a comparison between empirical mode decomposition (EMD) and smoothed pseudo Wigner-Ville distribution (SPWVD) methods based on vibration signature and energy calculation procedure for monitoring gearbox systems. The results showed that the calculation of energy using EMD techniques offers a more effective way to detect early faults than that computations using the SPWVD method, and that the computation of energy using the EMD technique is faster than the calculations done using the SPWVD method.

Adaptive noise cancelling is an effective technique for enhancing signal to noise ratio of corrupted signals. The effectiveness of the signal enhancement depends on the correlation degrees between primary signal and the reference signal. Many adaptive filter parameters search algorithms such as least mean square (LMS) algorithm, and recursive least squares (RLS) algorithm are used to remove a noise from signal [9].

This work presents a novel adaptive filtering scheme to the detection of damage in gears. The developed technique suits cases where a reference signal is not available; it is based on an-operating-condition dependent-artificially synthesized gearbox vibration signal, which can be assumed as the desired signal by which to extract a gear meshing signal from main signal via adaptive noise cancelling.

This paper is organized as follows: Adaptive noise cancelling and least mean squares algorithms are presented in Sections 2 and 3, respectively. The proposed scheme and numerical validation are illustrated in Section 4. Experimental work is presented in Section 5. The developed monitoring scheme is applied for detecting gear-tooth breakage in Section 6. Conclusions are outlined in Section 7.

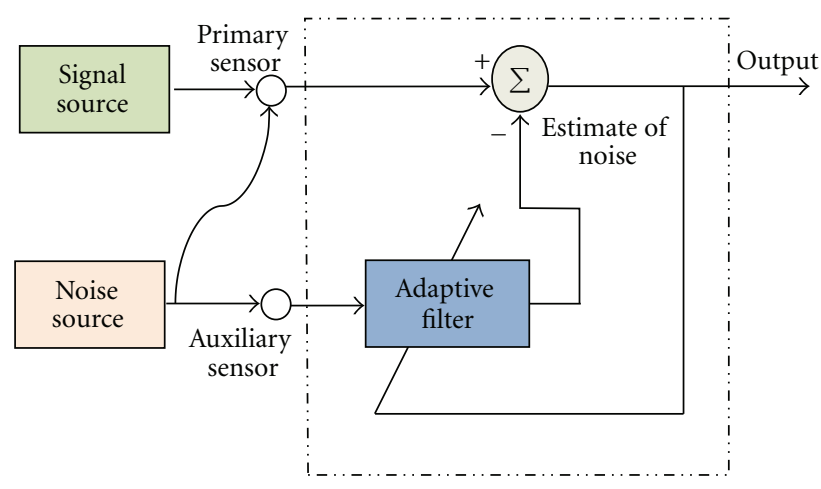

FIGURE 1: Schematic of adaptive noise cancellation system.

\section{Adaptive Filtering and Noise Cancellation}

One technique to improve the signal-to-noise ratio is adaptive noise cancelling. This is a technique whereby noise is subtracted from the original signal. Separate from the transducer detecting, the primary signal is a second or auxiliary transducer placed in a position where it is subject to much the same noise as the primary transducer but where the primary signal is very weak. The noise may now be eliminated by a process of subtraction. The problem is, of course, that the noise picked up by the auxiliary transducer will not usually exactly match the noise picked up by the primary transducer with the risk that part of the original signal may be lost. However, the risk of distorting the original signal (or decreasing signal-to-noise ratio) is substantially reduced if the noise signal to be subtracted is treated by a suitable filtering and adaptive process [10].

Figure 1 shows the essential components of an adaptive noise cancellation system: a dual-input, closed-loop, and adaptive feedback system [11].

The desired signal from the primary sensor $s(n)$ is contaminated with noise $N_{o}(n)$ which is assumed to be uncorrelated with $s(n)$. The combined signal from the primary sensor into the cancellation device is $\left(s(n)+N_{o}(n)\right)$. The auxiliary transducer receives noise $N_{1}(n)$ which is also assumed uncorrelated with the primary signal but correlated-though in an unknown way-with the noise $N_{o}(n)$. For example, both primary and secondary transducers might be microphones and pick-up airborne noise from a compressor. If the noise travels different distances to the two transducers there will be correlation between the two noise signals picked up by the microphones but the signals will differ in both phase and amplitude, and possibly have random differences due to gusting winds. It is usual to assume $s(n), N_{o}(n), N_{1}(n)$, and $y(n)$ are statistically stationary with zero means.

The noise signal $N_{1}(n)$ is filtered so that it is as close a replica as possible to $N_{o}(n)$. This filtered signal, $y(n)$, is subtracted from the combined signal to produce the output [12]:

$$
e(n)=s(n)+N_{o}(n)-y(n) .
$$


In Figure 1 the input from the auxiliary sensor is treated by an adaptive filter, which responds to an error signal that depends on the system output and automatically adjusts its impulse response until the closest fit between $e(n)$ and $s(n)$ is obtained. With noise cancellation the adaptive filter usually uses a least mean squares algorithm (LMS) to minimize the error in the output signal until $e(n)=s(n)$.

\section{Least Mean Squares Algorithms}

The LMS algorithm is a useful tool used with which to find optimal filter coefficients in real time. Its two great advantages are its simplicity and its almost universal use. The core of the LMS algorithm is the gradient search technique whereby an initial estimate is made of the coefficient vector, which is then iteratively updated so that it changes from its current estimate to a better estimate of the optimal filter coefficients [13].

Because $R$ and $P$, which appear in the Wiener-Hopf equation, are not known in advance and are time variant, the Wiener-Hopf equation is not usually immediately suitable for real-time applications. If higher filter orders are necessary then the inversion of $R$ can be very time consuming. The LMS algorithm is a means for calculating $W_{\text {opt }}$ without having to know either $R$ or $P$, and because it does not involve matrix inversion it is thus much more practical to use in real time. The LMS seeks to minimise the running average of the squared error signal $\left(e^{2}\right)$ rather than the expectation value $E\left[e^{2}\right]$. The LMS performs iterative calculations to determine the next set of Finite duration Impulse Response (FIR) coefficient, $w(n+1)$, from the values at step $n$. The speed of convergence of the LMS algorithm is controlled by introducing a factor $m$ which controls the step width of the iteration [14].

\section{The Proposed Scheme and Numerical Validation}

This section describes how LMS algorithms were applied to a signal simulated from gearbox frequencies. A sinusoidal approximation is used to represent a gear mesh signal (GMS) as [15]:

$$
\operatorname{GMS}(N, \theta)=\sin (N \times \theta),
$$

where $N$ represents the number of gear teeth, and $\theta$ the degree of shaft rotation.

The sidebands around the fundamental gear mesh frequency in the frequency domain are defined as sum and difference of the mesh frequency and gear or pinion speed [16]:

$$
\begin{aligned}
& f_{s p}=f_{m} \pm f_{p}, \\
& f_{s g}=f_{m} \pm f_{g} .
\end{aligned}
$$

With a gearbox operating at shaft frequency $22.53 \mathrm{~Hz}$, the gear meshing frequency at the first stage is $766.1 \mathrm{~Hz}$, and the

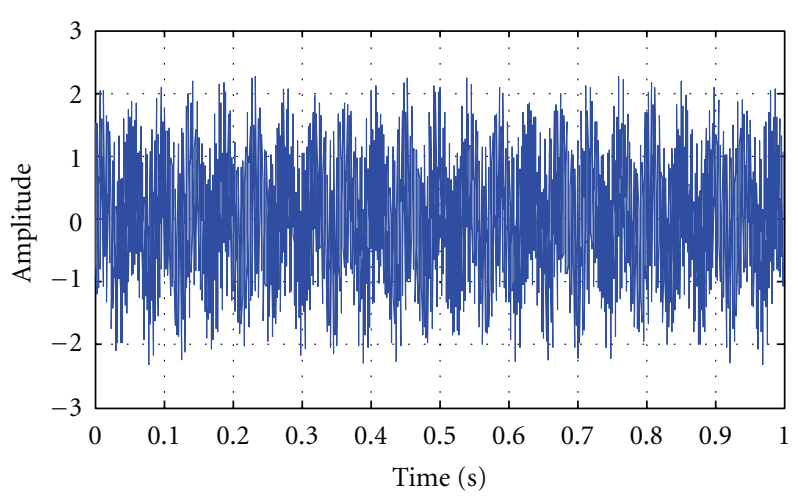

Figure 2: Time domain of simulated signal.

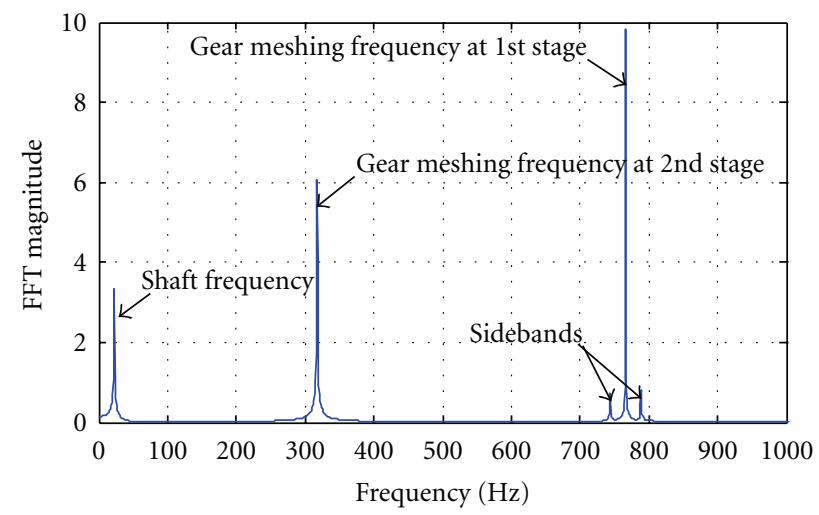

FIgURE 3: Frequency domain of simulated signal.

gear meshing frequency at the second stage is $317.4 \mathrm{~Hz}$, a simulated signal can be assumed to be:

$$
\begin{aligned}
x(t)= & 0.5 * \sin (2 * \pi * 22.53 * t) \\
& +\sin (2 * \pi * 766.1 * t) \\
& +0.8 * \sin (2 * \pi * 317.4 * t) \\
& +0.1 * \sin (2 * \pi * 788.63 * t) \\
& +0.1 * \sin (2 * \pi * 743.57 * t) .
\end{aligned}
$$

Figures 2 and 3 represent time domain and FFT, respectively for signal $x(t)$.

Figure 3 shows sidebands around the 1st-stage gear meshing frequency at frequencies of $743.57 \mathrm{~Hz}(766.1-$ $22.53)$ and $788.63 \mathrm{~Hz}(766.1+22.53)$. In a real operating gearbox there will be different noises from different sources and the sidebands will be affected. To understand the effect of noise on a sideband let signal $x(t)$ be corrupted by random noise as shown in Figure 4. The FFT of the corrupted signal appears in Figure 5 and it can be clearly seen that the sidebands are no longer discernible.

An LMS algorithm was applied to remove noise from signal and the results can be seen in Figures 6 and 7 which also show the effects of the step size chosen. To examine step size of LMS on signal, two values of step size were chosen 


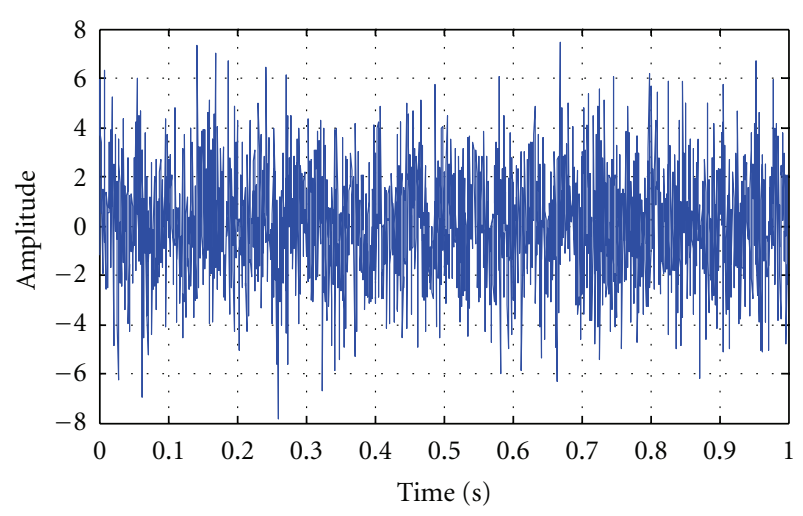

FIGURE 4: Time domain of simulated corrupted signal.

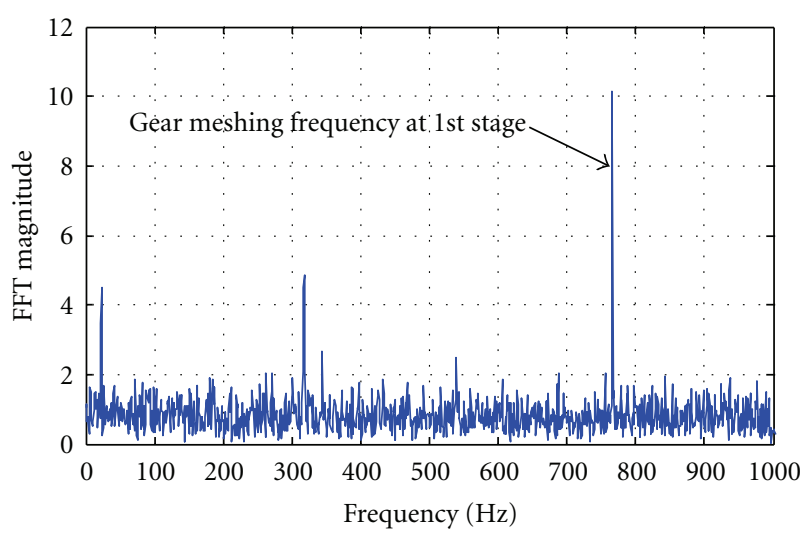

FIGURE 5: Frequency domain for simulated corrupted signal.

for the simulation: 0.0001 and 0.00001 . Figure 6 shows the adaptive noise cancelling applied to the simulated corrupted signal to remove noise with step size of 0.0001 . It can be seen that there is an improvement in the signal with the sidebands beginning to appear either side of gear meshing frequency. Figure 7 shows the results when the step size was 0.00001 . Clearly there has been a substantial improvement in signal clarity (much greater signal-to-noise ratio) and the sidebands are now clearly discernible.

\section{Experimental Setup}

The experimental data were collected using the test rig shown in Figure 8. It comprises a two-stage, $11 \mathrm{Kw}$, helical gearbox drive by three-phase induction motor and connected to a DC generator and adjacent resistor banks.

Test were carried out using healthy pair of gears and one pair suffering from a tooth breakage with magnitude fault 1 ( $25 \%$ tooth removal), fault 2 (50\% tooth removal), and fault 3 (75\% tooth removal) of the tooth under varying loads $(0 \%, 20 \%, 40 \%, 60 \%$, and $80 \%)$. The drive pinion at the first stage had 34 teeth meshing with a 70-tooth wheel. The pinion gear at the second stage had 29 teeth meshing with 52 -tooth wheel. The vibration signals were collected using an accelerometer mounted vertically on the gearbox housing.

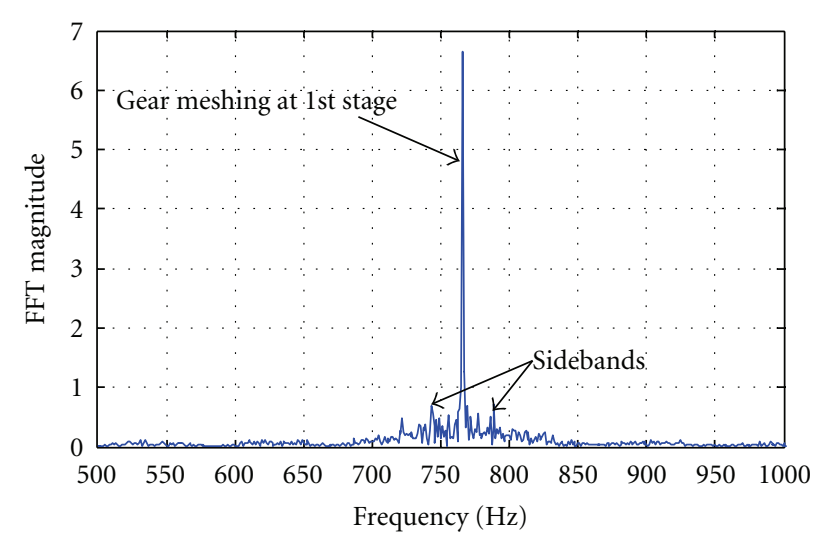

FIGURE 6: Frequency domain of corrupted signal obtained using noise cancellation with LMS step size 0.0001 .

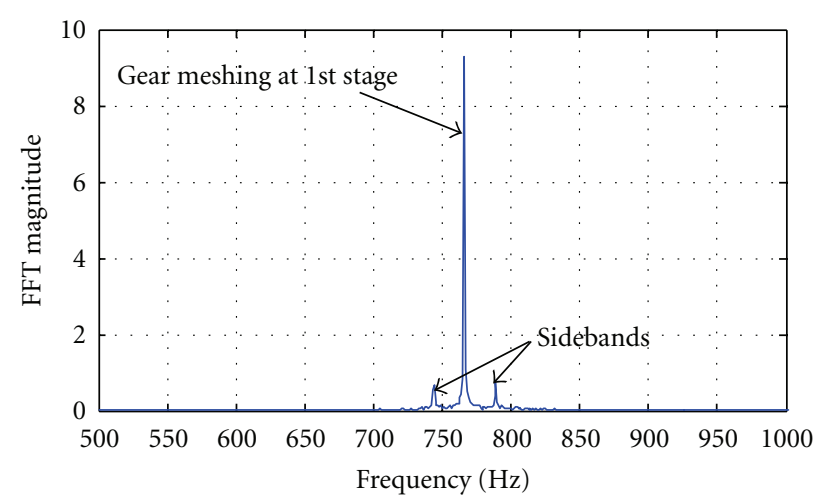

FIGURE 7: Frequency domain of corrupted signal obtained using noise cancellation with LMS step size 0.00001 .

The accelerometers were B\&K type 4371 with sensitivity of $10 \mathrm{mV} / \mathrm{g}$ and suitable for vibration measurements within a range of $1 \mathrm{~Hz}$ to $12 \mathrm{kHz}$. The sampling frequency was set to $50 \mathrm{kHz}$. Before the accelerometer signals were fed to the analogue-to-digital converter NI USB 9233 card, they passed through a B\&K type 2635 charge amplifier to condition the signal. The charge amplifier converts the accelerometer high impedance, low charge (in the range of Pico-coulomb) signal into low impedance and high voltage (in the range of $\mathrm{mV}$ ) and the cut off frequency for initialising filter was set to $10 \mathrm{kHz}$.

\section{Gear Fault Features Enhancement}

This section reports the application of the LMS algorithm to the detection of gear-tooth breakage. The data sets measured under operating conditions of: no fault, $25 \%$ tooth removal, and $50 \%$ tooth removal, carries many sinusoidal waveforms of different frequencies. A sinusoidal approximation was assumed as the desired signal and used to extract the gear 


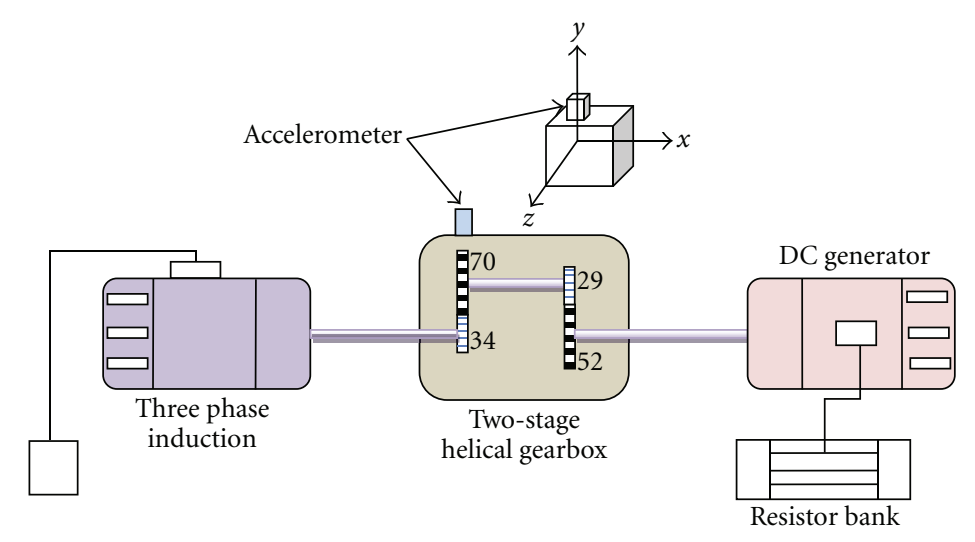

Figure 8: Schematic layout of the gearbox test rig.

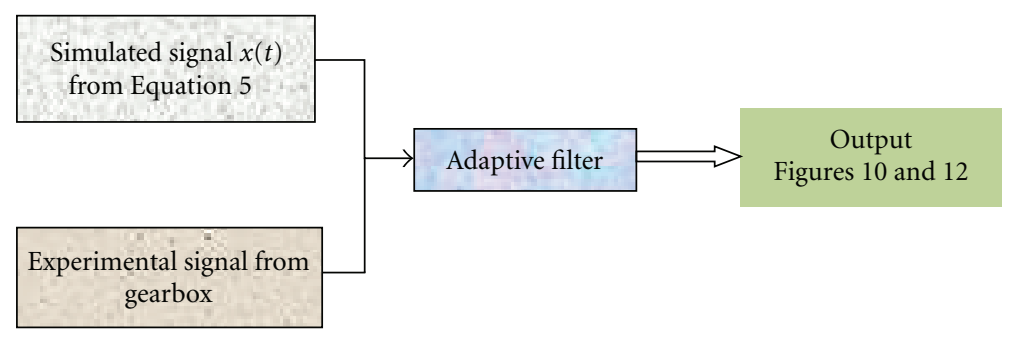

FIGURE 9: The proposed scheme architecture.

meshing signal from main signal. A desired signal can be expressed as:

$$
\begin{aligned}
x(t)= & \frac{\mathrm{RMS}(\text { main signal })}{3} * \sin \left(2 * \pi * f_{s} * t\right) \\
& +\mathrm{RMS}(\text { main signal }) * \sin \left(2 * \pi * f_{m 1} * t\right) \\
& +\frac{\mathrm{RMS}(\text { main signal })}{2} * \sin \left(2 * \pi * f_{m 2} * t\right) \\
& +\frac{\mathrm{RMS}(\text { main signal })}{3} * \sin \left(2 * \pi * f_{\text {sidel }} * t\right) \\
& +\frac{\mathrm{RMS}(\text { main signal })}{3} * \sin \left(2 * \pi * f_{\text {side2 }} * t\right),
\end{aligned}
$$

where RMS(main signal) is the root mean square of the main signal, $f_{s}$ is shaft frequency, $f_{m 1}$ is gear meshing frequency at 1 st stage, $f_{m 2}$ is gear meshing frequency at 2 nd stage and $f_{\text {sidel }}, f_{\text {side2 }}$ are sidebands frequencies.

Vibration signals collected from the gearbox housing under no load and $80 \%$ of the total load was considered as reference when using the LMS algorithm. Figure 9 illustrates the proposed scheme architecture for simulated and experimental signals.

Figures 10 and 12 show the vibration signal in frequency domain, under no load and $80 \%$ of the total load, after applying the LMS algorithm. Figures 11 and 13 zoom in on the upper sideband. The FFT magnitude is $5.8,7$, and 10.1 for healthy, $25 \%$ tooth removal and $50 \%$ tooth removal,

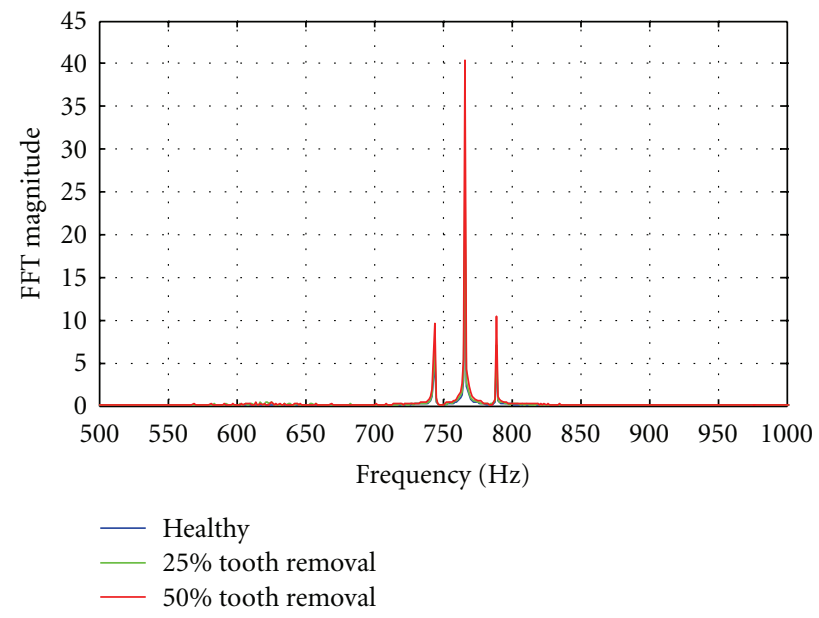

Figure 10: Frequency domain of gear vibration obtained after applying LMS algorithm (zero load).

respectively, under zero load; and it is 33, 40, and 48 for healthy, $25 \%$ tooth removal and 50\% tooth removal, respectively, under $80 \%$ of total load. From these figures, it is clear that there is a relationship between the fault level and the amplitude of sidebands for all cases considered. The amplitude of the sidebands increased as the damage level increased. It is concluded that there is advantage to applying the LMS algorithm for fault detection. 


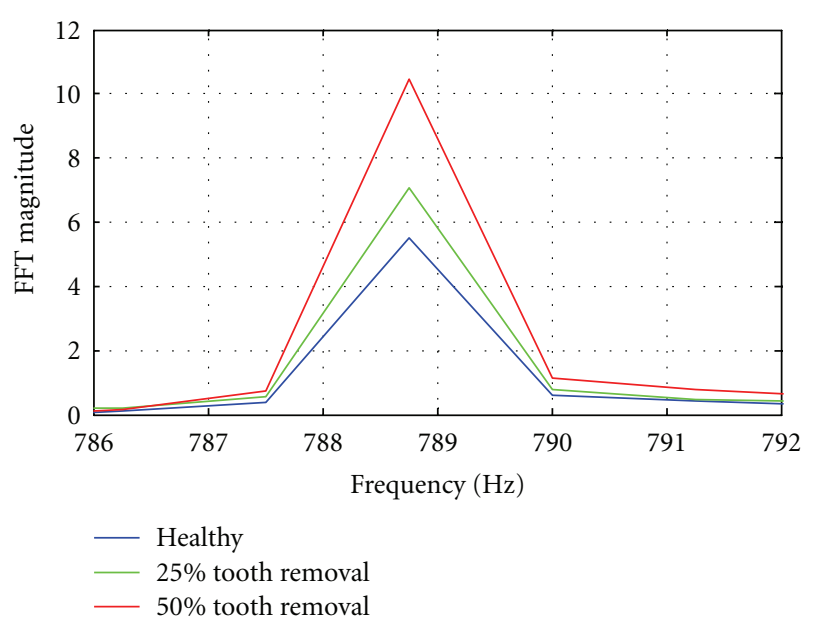

Figure 11: Zoom onto sideband (zero load).

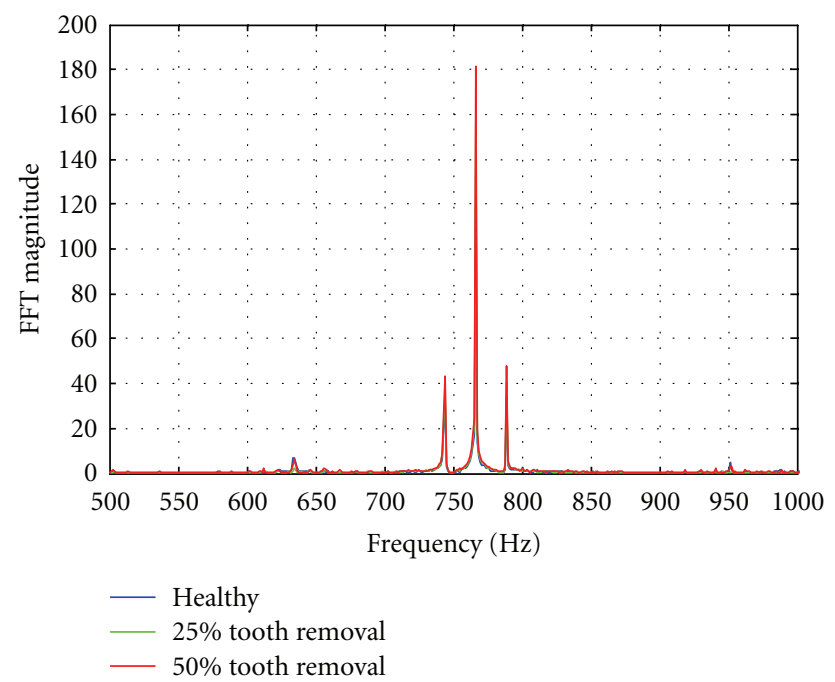

FIGURE 12: Frequency-domain of gear vibration obtained after applying LMS algorithm ( $80 \%$ of total load).

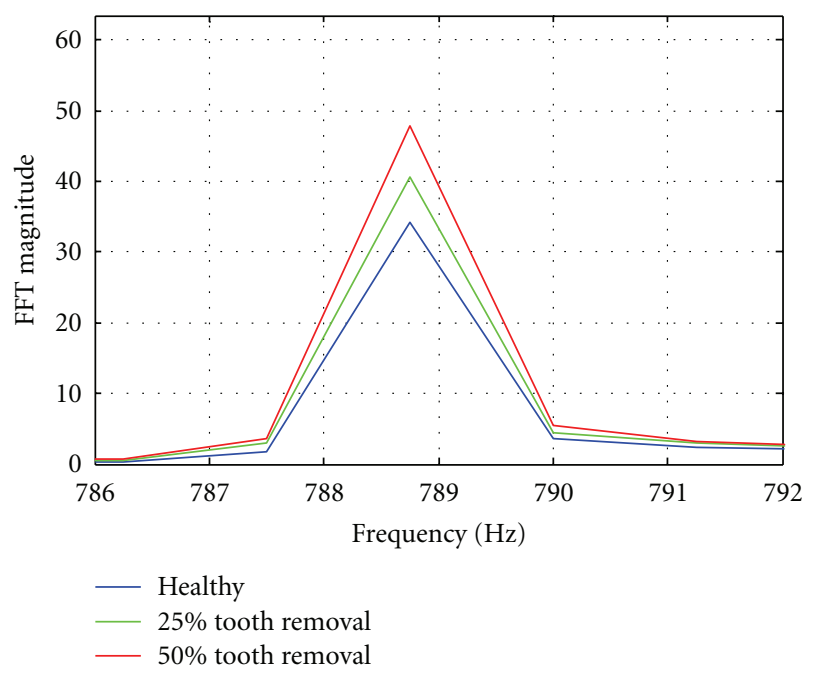

FIgURE 13: Zoom onto sideband (80\% of total load).

\section{Conclusion}

To improve signal-to-noise ratio the LMS algorithm was adopted, modified, and applied to signals which simulated gearbox frequencies and signals collected experimentally from a gearbox. The novelty lies on using only one accelerometer as the main signal and a desired signal was artificially generated from the measured shaft and gear meshing frequencies. The conclusions can be summarized as follows.

(1) A sinusoidal approximation (5) was used as the desired signal to extract the gear meshing signal from the main signal.

(2) The new proposed procedure was effective at revealing faults by producing larger amplitude sidebands as shown Figures 11 and 13.

(3) All previous researchers used at least two accelerometers in adaptive filtering but in this work one accelerometer was used and the results appeared good, which could mean reduced monitoring costs.

\section{References}

[1] G. Liao, S. Liu, T. Shi, and G. Zhang, "Gearbox condition monitoring using self-organizing feature maps," Proceedings of the Institution of Mechanical Engineers, Part C, vol. 218, no. 1, pp. 119-130, 2004.

[2] Q. He, F. Kong, and R. Yan, "Subspace-based gearbox condition monitoring by kernel principal component analysis," Mechanical Systems and Signal Processing, vol. 21, no. 4, pp. 1755-1772, 2007.

[3] J. Rafiee and P. W. Tse, "Use of autocorrelation of wavelet coefficients for fault diagnosis," Mechanical Systems and Signal Processing, vol. 23, no. 5, pp. 1554-1572, 2009.

[4] Y. Zhou, T. Tao, X. Mei, G. Jiang, and N. Sun, "Feed-axis gearbox condition monitoring using built-in position sensors and EEMD method," Robotics and Computer-Integrated Manufacturing, vol. 27, no. 4, pp. 785-793, 2011.

[5] B. Eftekharnejad and D. Mba, "Seeded fault detection on helical gears with acoustic emission," Applied Acoustics, vol. 70, no. 4, pp. 547-555, 2009.

[6] N. Saravanan, V. N. S. K. Siddabattuni, and K. I. Ramachandran, "Fault diagnosis of spur bevel gear box using artificial neural network (ANN), and proximal support vector machine (PSVM)," Applied Soft Computing Journal, vol. 10, no. 1, pp. 344-360, 2010.

[7] A. Albarbar, F. Gu, A. D. Ball, and A. Starr, "Acoustic monitoring of engine fuel injection based on adaptive filtering techniques," Applied Acoustics, vol. 71, no. 12, pp. 1132-1141, 2010.

[8] G. R. Ibrahim and A. Albarbar, "Comparison between Wigner-Ville distribution- and empirical mode decomposition vibration-based techniques for helical gearbox monitoring," Proceedings of the Institution of Mechanical Engineers, Part C, vol. 225, no. 8, pp. 1833-1846, 2011.

[9] X. Zhou, Y. Shao, D. Zhen, F. Gu, and A. Ball, "Gear fault signal detection based on an adaptive fractional Fourier transform filter," Journal of Physics, vol. 305, no. 1, Article ID 012022, 2011.

[10] B. Widrow, J. R. Glover, and J. M. McCool, "Adaptive noise cancelling: principles and applications," Proceedings of the IEEE, vol. 63, no. 12, pp. 1692-1716, 1975. 
[11] S. Haykin, Adaptive Filter Theory, 3rd edition, 1995.

[12] M. Abdulmagid, D. Krusienski, S. Pal, and W. Jenkins, "Principles of adaptive noise cancelling," Annual Research Journal, vol. 2, 2004.

[13] V. Bajic, Design and implementation of an adaptive noise canceling system in wavelet transform domain [M.S. thesis], University of Akron, 2005.

[14] W. Fohl, J. Matthies, and B. Schwarz, "A FPGA-based adaptive noise cancelling system," in Proceedings of the 12th International Conference on Digital Audio Effects (DAFx '09), Como, Italy, 2009.

[15] C. J. Stander and P. S. Heyns, "Instantaneous angular speed monitoring of gearboxes under non-cyclic stationary load conditions," Mechanical Systems and Signal Processing, vol. 19, no. 4, pp. 817-835, 2005.

[16] Barron, Engineering Condition Monitoring Practice, Methods and Application, Addison-Wesley/Longman, New York, NY, USA, 1996. 

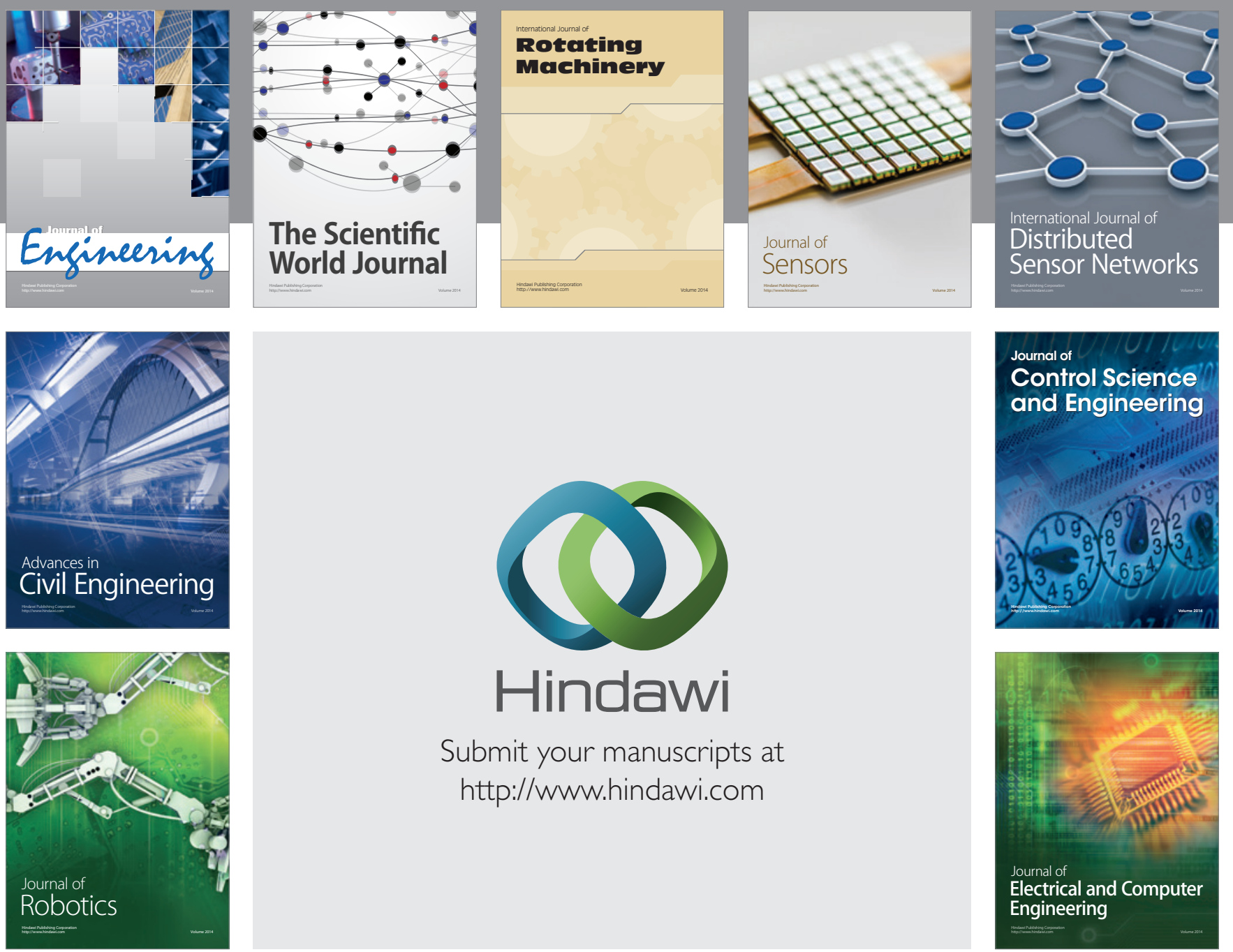

Submit your manuscripts at

http://www.hindawi.com
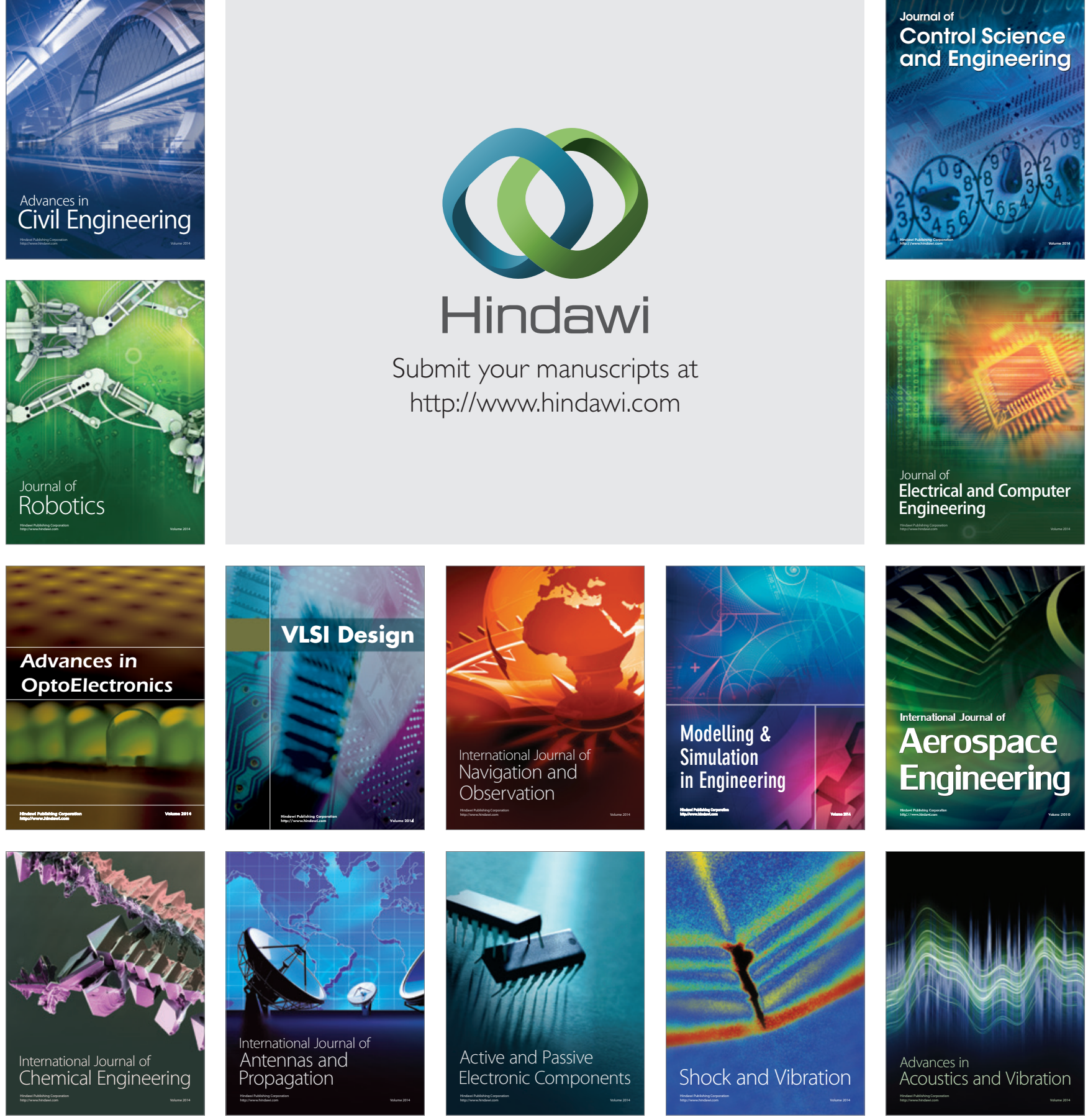